\title{
«Smarter Medicine»: 5 Interventionen, die in der ambulanten allgemeinen inneren Medizin vermieden werden sollten
}

K. Selby ${ }^{a}$, J. Cornuz ${ }^{a}$,

S. Neuner-Jehle ${ }^{b}$, A. Perrier ${ }^{c}$,

A. Zellerd, C. A. Meiere,

N. Rodondif, J. M. Gaspoz ${ }^{g}$

a Policlinique médicale universitaire, Lausanne

b Institut für Hausarztmedizin Zürich

c Service de médecine interne générale, Hôpitaux Universitaires de Genève

d Institut für Hausarztmedizin, Basel

e Department Innere Medizin und Spezialdisziplinen, Stadtspital Triemli, Zürich

f Clinique Universitaire de Médecine Interne Générale, Hôpital de l'Ile,

Université de Berne

g Service de médecine de premier recours, Hôpitaux Universitaires de Genève

Korrespondenz: Kevin Selby

Policlinique médicale universitaire Rue du Bugnon 44 CH-1011 Lausanne

\section{Einleitung}

Seit 2012 befasst sich die Schweizerische Gesellschaft für Allgemeine Innere Medizin mit der Problematik der Überdiagnostik und Überversorgung in der Medizin. Nun hat sie beschlossen, eine Liste mit fünf Untersuchungen auf dem Gebiet der ambulanten allgemeinen inneren Medizin zusammenzustellen, die ohne oder mit nur geringem Nutzen bei zahlreichen Patienten durchgeführt werden, gleichzeitig jedoch unerwünschte Nebenwirkungen haben können und zum Anstieg der Gesundheitskosten beitragen.

\section{Vorgehen}

Eine genauere Version der angewandten Methodik wird demnächst veröffentlicht werden. Laut einer Auswertung der relevanten Literatur gibt es drei internationale Projekte, darunter die «Choosing wisely initiative» in den USA, die Interventionen auflisten, welche es im Interesse einer höheren Effizienz und einer besseren Qualität der Versorgung zu vermeiden gilt [1-3]. Auf dieser Basis wurde eine Vorauswahl von 38 Untersuchungen auf dem Gebiet der ambulanten allgemeinen inneren Medizin zusammengestellt. 35 Experten - Ärzte aus dem Bereich der allgemeinen inneren Medizin und Hausärzte - nahmen an einer elektronischen Delphi-Umfrage teil, bei der sie die internationalen Empfehlungen nach ihrer Priorität ordneten und um zwölf neue Empfehlungen ergänzten. Die 18 am höchsten eingestuften Interventionen wurden erneut geprüft und nach der Häufigkeit ihrer Anwendung in der Schweiz geordnet. Die zehn höchstplatzierten Interventionen wurden in einer Liste zusammengefasst. Ausgehend von den Empfehlungen der Experten wählte das SGIM-Komitee fünf Interventionen aus, die nun als erste Liste offizieller Empfehlungen vorgelegt wurden.

\section{Ergebnisse}

Die Schweizerische Gesellschaft für Allgemeine Innere Medizin empfiehlt, von folgenden Untersuchungen und Verordnungen abzusehen: 1. Durchführen bildgebender Diagnostik in den ersten 6 Wochen bei Patienten mit unspezifischen Lumbalgien. 33\% der Schweizer Bevölkerung > 15 Jahren leiden unter leichten Rückenschmerzen, 10\% unter schweren [4]. Seit mehreren Jahren gibt es internationale Empfehlungen, die eine konservative Versorgung nahelegen, wenn die Patientin oder der Patient keine Alarmsignale («red flags») wie gravierende oder fortschreitende neurologische Defizite aufweist und kein
Verdacht auf bösartige oder infektiöse Prozesse besteht. Dennoch kam es laut einer aktuellen Studie zwischen 2000 und 2010 zu einer vermehrten Anwendung neuer bildgebender Verfahren (CT und MRT) ohne gleichzeitigen Rückgang der konventionellen radiologischen Verfahren [5]. Die Anwendung eines bildgebenden Verfahrens innerhalb der ersten sechs Wochen bei unspezifischen Rückenschmerzen führt zu einem Anstieg der Gesundheitskosten, ohne die Schmerzen zu lindern oder das Risiko für eine Behinderung zu senken [6]. Vor allem mit der Verordnung einer MRT steigt das Risiko für einen chirurgischen Eingriff, ohne dass deshalb der Grad oder die Dauer der Schmerzen oder der Behinderung zurückgingen [7].

2. Messung des Prostata-spezifischen Antigens (PSA) zwecks Prostatakrebs-Screening ohne eine Diskussion von Risiko und Nutzen.

Jedes Jahr wird bei 25\% der Männer zwischen 50 und 74 Jahren in der Schweiz der PSA-Wert (prostate-specific antigen) bestimmt [4]. Zwei umfangreiche randomisierte Studien ergaben widersprüchliche Ergebnisse hinsichtlich des Nutzens dieser Messung. Die Risiken falsch-positiver Ergebnisse, einer Überdiagnostik und darauf folgender Untersuchungen und Behandlungen sind erheblich [8]. Fast sämtliche aktuellen Richtlinien empfehlen die Durchführung des PSA-Tests ausschliesslich nach hinreichender Aufklärung des Patienten über die Gefahren eines falsch-positiven Ergebnisses und die begrenzte Aussagekraft des Tests. Die Aufklärung sollte in Form eines strukturierten Gesprächs erfolgen, bei dem eine gemeinsame Entscheidung getroffen wird. Patienten über 75 Jahren sollte der Test nicht mehr angeboten werden. Dennoch fand in den USA bei nur 13\% der Männer, die sich einem PSA-Test unterzogen, eine umfassende Aufklärung über die Vor- und Nachteile und die Unsicherheiten eines PSA-Screenings statt [9].

3. Verschreiben von Antibiotika gegen unkomplizierte Infekte der oberen Luftwege.

In Grossbritannien konsultiert jährlich ein Viertel der Bevölkerung aufgrund eines Infekts der oberen Atemwege den Hausarzt. 60\% der Antibiotika werden zur Behandlung von Atemwegsinfekten verordnet [10]. Man weiss jedoch, dass die Mehrzahl dieser Infekte viralen Ursprungs ist. Selbst eitriger Auswurf ist kein Anzeichen für eine bakterielle Ursache. Die Symptome eines Infekts der oberen Atemwege halten 7 bis 11 Tage an. Die Einnahme von Antibiotika verkürzt diese Dauer nicht und erhöht vielmehr die Gefahr von Resistenzen [11]. Auf 
die Symptome zugeschnittene Verordnungen und eine spezifische Beratung scheinen die Patienten ebenso zufriedenzustellen wie die Verordnung von Antibiotika [10].

4. Durchführen eines präoperativen Thorax-Röntgenbildes, ausser bei Verdacht auf intrathorakale Pathologie. Jahr für Jahr werden in der Schweiz über eine Million chirurgischer Eingriffe vorgenommen. Meist wird im Rahmen der präoperativen Untersuchungen auch ein thorakales Röntgenbild angefordert. Nur $2 \%$ der bei asymptomatischen Patienten angefertigten Röntgenbilder ändern etwas an der folgenden medizinischen Versorgung. Es gibt keine Studie, die belegt, dass ein thorakales Röntgenbild zusätzliche Informationen liefert, die über die Anamnese und die körperliche Untersuchung hinausgehen, oder dass es für den Patienten von Nutzen ist. Es existieren keine Daten, die bestätigen, dass eine radiologische Basis-Abklärung bei Komplikationen zu einer anderen Versorgung führt, ausgenommen bei älteren Patienten (70+) mit vorbestehender Lungenerkrankung [12].

5. Weiterführen einer Langzeit-Pharmakotherapie bei gastrointestinalen Symptomen mit ProtonenPumpenblockern ohne Reduktion auf die tiefste wirksame Dosis.

Die Verordnungshäufigkeit von Protonenpumpenhemmern nimmt derzeit deutlich zu. Im Kanton Genf zum Beispiel hat der Konsum von PPI zwischen Anfang 2000 und Ende 2008 um fast 500\% zugelegt [13]! PPI reduzieren wirksam rezidivierende Refluxsymptome und dienen der Behandlung akuter Magendarmgeschwüre. Ausserhalb von Indikationen, die auf einem pathologischen Endoskopiebefund basieren, haben sie keinerlei präventiven Nutzen [14]. Dagegen kann die chronische Behandlung mit PPI zu einem Anstieg des Risikos für osteoporotische Frakturen, Pneumonien, Clostridium difficileInfektionen, interstitieller Nephritis und Vitamin-B12-Mangel führen [13]. Dieses Risiko nimmt dosisabhängig zu. Die Notwendigkeit einer Langzeitbehandlung muss daher sorgfältig geprüft werden. Bei häufigen Beschwerden ist gegebenenfalls die tiefste wirksame Dosis zu verordnen. Bei wiederkehrenden Symptomen ist eine intermittierende Einnahme oder die Verordnung eines anderen Medikaments zu erwägen.

\section{Schlussfolgerung}

Diese erste Auflistung von Untersuchungen und Verordnungen, die vermieden werden sollten, ist Teil des ehrgeizigen «Smarter Medicine»-Projekts der SGIM (www.smartermedicine.ch), dessen Ziel es ist, die Qualität der Versorgung zu verbessern und dabei die Entscheidungsautonomie von Arzt und Patient zu wahren. Sie soll zur Reflektion über die Überdiagnostik und Überversorgung in der Medizin beitragen und das Konzept einer gemeinsamen Entscheidungsfindung von Arzt und Patient unterstützen.

1 American Board of Internal Medicine Foundation. Choosing Wisely. 2013. www.choosingwisely.org/

\section{smartermedicine}

Weniger Medizin kann mehr sein

Die «Smarter Medicine»-Kampagne - ein sinnvolles und nachhaltiges Engagement zum Wohl der Patienten in der Schweiz

Die Schweizerische Gesellschaft für Allgemeine Innere Medizin (SGIM) lanciert als Pionierin für die Schweiz am 14. Mai 2014 im Rahmen des ESCIM2014-Kongresses in Genf erstmals eine Liste mit Abklärungen und Therapien im Rahmen der Allgemeinen Inneren Medizin, die keinen messbaren Nutzen für den Patienten darstellen und bei denen das Risiko den potenziellen Nutzen möglicherweise übersteigt. Die Liste bildet die Basis der geplanten SGIMKampagnenaktivitäten «Smarter Medicine». Diese in der Schweiz einmalige Kampagne, die auch Forschungstätigkeiten, z. B. im Rahmen der «Choosing wisely»-Preisausschreibung 2013/2014 der SGIMFoundation, umfasst, orientiert sich an den Vorgaben einer effizienten und evidenzbasierten Medizin sowie am Positionspapier zum Thema «Ein nachhaltiges Gesundheitssystem für die Schweiz» der Schweizerischen Akademie für Medizinische Wissenschaften (SAMW). www.smartermedicine.ch

2 National Institute of Health and Clinical Excellence. NICE «do not do» recommendations. 2013. www.nice.org.uk/ usingguidance/donotdorecommendations/

3 Elshaug AG, Watt AM, Mundy L, Willis CD. Over 150 potentially low-value health care practices: an Australian study. The Medical Journal of Australia. 2012;197:556-60.

4 David Altwegg et al. Statistiques de la santé 2012. Neuchâtel: Office fédéral de la statistique; 2012.

5 Mafi JN, McCarthy EP, Davis RB, Landon BE. Worsening trends in the management and treatment of back pain. JAMA internal medicine. 2013;173:1573-81.

6 Chou R, Fu R, Carrino JA, Deyo RA. Imaging strategies for low-back pain: systematic review and meta-analysis. Lancet. 2009;373:463-72.

7 Jarvik JG, Hollingworth W, Martin B, et al. Rapid magnetic resonance imaging vs radiographs for patients with low back pain: a randomized controlled trial. JAMA. 2003;289:2810-8.

8 Moyer VA, USPST. Screening for prostate cancer: U.S. Preventive Services Task Force recommendation statement. Annals of internal medicine. 2012;157:120-34.

9 Han PK, Kobrin S, Breen N, et al. National evidence on the use of shared decision making in prostate-specific antigen screening. Annals of family medicine. 2013;11:306-14.

10 Centre for Clinical Practice. Respiratory tract infections - antibiotic prescribing. London: National Institute for Health and Clinical Excellence; 2008

11 Gonzales R, Bartlett JG, Besser RE, et al. Principles of appropriate antibiotic use for treatment of nonspecific upper respiratory tract infections in adults: background. Annals of internal medicine. 2001;134:490-4.

12 Mohammed TL, Kirsch J, et al. ACR Appropriateness Criteria routine admission and preoperative chest radiography. Reston: American College of Radiology; 2011.

13 Roulet L, Vernaz N, Giostra E, Gasche Y, Desmeules J. [Adverse effects of proton pump inhibitors: should we worry about long-term exposure?]. La Revue de médecine interne / fondee par la Société nationale française de médecine interne. 2012;33:439-45.

14 Kahrilas PJ, Shaheen NJ, Vaezi MF, et al. American Gastroenterological Association Institute technical review on the management of gastroesophageal reflux disease. Gastroenterology. 2008;135:1392-413, 413 e1-5. 